\title{
SOME PROBLEMS OF META-GALACTIC RADIO-EMISSION
}

\author{
I. S. SHKLOVSKY \\ Sternberg Astronomical Institute, Moscow, U.S.S.R.
}

The observed cosmical radio-emission has to be divided into the galactic and metagalactic components. The separation of these components is a problem of first importance. It was shown by us (Shklovsky, I952 [1]) that at least $75 \%$ of the observed intensity near the galactic poles is caused by sources located in our stellar system. These sources form an almost spherical sub-system. Another model of the distribution of the cosmical radio-emission sources (Westerhout and Oort, I95 [2]) is popular in the western countries. The so-called 'isotropic component' of metagalactic origin used in that model was considered responsible for the greatest part of the intensity near the galactic poles.

Recent observations of the distribution of the intensity of radio-emission in $\mathrm{M}_{3} \mathrm{I}$ (Baldwin, I954 [3]) give a new and most important argument in favour of our model of the distribution of the sources of cosmical radioemission. The temperature of the metagalactic emission at $\lambda=3$ metres thus lies between $100^{\circ}$ and $200^{\circ}$.

Optical observations do not permit us to estimate the integral brightness of the Meta-Galaxy, because the faint light from the latter is lost in the much brighter glow of atmospheric, interplanetary and galactic origin. The fundamental cosmological characteristics entering into the theory of the integral photometric effect of the Meta-Galaxy are the average density and the red-shift constant. This demonstrates the decisive importance of radioastronomical methods in cosmology, which was underlined by us in $1953^{[4]}$. It was proved (Shklovsky, I953 [4]) that the phenomenon of the red-shift exists also for the meta-galactic radio-emission. The so-called ' photometric radius of the Meta-Galaxy', i.e. the radius of an imaginary sphere of constant density, in which the radio sources (similar to those in the surroundings of our Galaxy), when uniformly distributed, give a radio intensity equal to the observed, was estimated. This radius was found about 500 megaparsecs (on the new scale), which confirms the theoretical calculations, based upon relativistic cosmology. 
Radio-emission from several clusters of galaxies and the so-called 'Super-Galaxy' was discovered recently. The question arises whether this radio-emission is caused by all 'radio galaxies' contained in this cluster (the 'discrete' hypothesis) or localized in the intergalactic space within the cluster ('continuous' hypothesis). The 'discrete' hypothesis was until now accepted by the majority of investigators. However, we have shown that this hypothesis meets considerable difficulties (Shklovsky, 1954[5]) and that the 'continuous' hypothesis is more acceptable. If radio-emission is caused by relativistic electrons in magnetic fields, then, according to our estimates, the concentration of the relativistic electrons in metagalactic space should be of the order of $10^{-13} \mathrm{~cm}^{-3}$ and the strength of the magnetic field $\mathrm{H} \sim \mathrm{IO}^{-7}$ gauss. This also gives us a new aspect for the problem of the origin of cosmic rays.

The inter-galactic medium in clusters of galaxies may also cause the radio line of $21 \mathrm{~cm}$. The frequency of this emission from the given cluster will be strongly shifted and will be concentrated in a wide band of the order of $\mathrm{I}$ to $2 \mathrm{Mc}$./s. The brightness temperature may reach several tens of degrees. Special instruments are being developed in the Moscow university for the solution of this most important problem.

Up to 2000 discrete sources of $F_{\nu}>7 \cdot 5 \cdot 10^{-26}$ watt per m..$^{2}$ per herz $(\lambda=3.7$ metres) are known at present. The majority of them are of a metagalactic origin. The question arises to what type of radio galaxies the majority of these sources must be identified. What causes the observed radio intensity from the Meta-Galaxy: clusters of galaxies or discrete sources? The total flux emitted by all the known discrete sources equals about $5 \cdot 10^{-22}$ watt per m. ${ }^{2}$ per herz, which corresponds to an average brightness temperature of about $15^{\circ}(\lambda=3.7$ metres $)$, or about $5 \%$ of the total brightness of the Meta-Galaxy.

If these faintest sources should be similar to Cygnus A then it may be found by means of methods used in stellar astronomy that their spatial density should be $10^{8}$ times smaller than the density of late-type spirals. The mean distance between them should be of the order of 300 megaparsecs and the faintest of them should be located at an enormously large distance of $4.10^{9}$ parsecs. However, we did not take into account the red shift, which will cut off the emission of the objects, located at distances over 800 megaparsecs, rather strongly. Consequently, unless recourse is taken to very special cosmological models, the faintest of the known sources can hardly be identified with objects like Cygnus A. If the sources should be identified with objects like $\mathrm{NGC}_{4486}$ then their spatial density should constitute about $\mathrm{I} / \mathrm{I}$ oo of the spatial density of late spirals. The sources 
with $F_{\nu}=7.5 \times 10^{-26}$ watt per m. ${ }^{2}$ per herz should then belocated at distances of about 80 megaparsecs. But while the 'photometric radius' of the MetaGalaxy equals 500 megaparsecs, these sources, located in different parts of the Meta-Galaxy, will produce only about $35 \%$ of the observed surface brightness of the Meta-Galaxy. This may signify that the brightness of the Meta-Galaxy is determined to a considerable extent by the integral effect of clusters of galaxies.

Finally, if the faintest known sources are identified with radio galaxies of comparatively low luminosity of the type of $\mathrm{NGG}_{5} \mathrm{I}_{28}$ or $\mathrm{NGG}_{13}{ }_{16}$, the partial density in respect to the late spirals must be about $1 / 10$. The main part of the observed brightness of the Meta-Galaxy can be explained by the integral radiation from these sources.

In this connexion attention may be paid to the fact that from seventeen galaxies brighter than ${ }_{10}{ }^{m}$, three $\left(\mathrm{NGC}_{5128,4486}\right.$ and $\left.{ }_{13}{ }^{16} 6\right)$ are radio galaxies. This may signify that these three belong to a class of objects met comparatively often in the Meta-Galaxy. If the majority of faint discrete sources are considered similar to $\mathrm{NGG}_{5128}$ and $\mathrm{NGG}_{13}{ }_{16} 6$, then these sources should be identified with galaxies of about 14 to $15^{\mathrm{m}}$. If the faint discrete sources are caused by objects of the type of $\mathrm{NGC}_{44} 86$ they must be identified with galaxies of about $16^{\mathrm{m}}$. The total number of such galaxies in the sky is about $10^{5}$. Thus, the problem of identification of metagalactic discrete sources with galaxies becomes extremely important.

\section{REFERENGES}

[1] Shklovsky, I. S. A.J. U.S.S.R. 29, 418, 1952.

[2] Westerhout, G. and Oort, J. H. B.A.N. 11, 323, no. 426, I951.

[3] Baldwin, J. Nature, 74, 320, 1954.

[4] Shklovsky, I. S. A.J. U.S.S.R. 30, 495, 1953.

[5] Shklovsky, I. S. A.J. U.S.S.R. 31, 533, 1954. 